\title{
Diagnóstico e notificação de casos de violência contra crianças e adolescentes: conhecimento de estudantes de Odontologia
}

\author{
Thaís Farias Alvarenga da Hora Nunes*; Letícia Santos Alves de Melo*; Viviane Colares**; Alice \\ Kelly**** \\ * Cirurgiã-dentista graduada pela UFPE \\ ** Professora Adjunta, Departamento de Clínica e \\ Odontologia Preventiva, UFPE; Professora Associada, \\ Faculdade de Odontologia da UPE \\ *** Professora Adjunta, Departamento de Clínica e \\ Odontologia Preventiva, UFPE
}

Recebido em: 26/08/2019. Aprovado em: 21/04/2021.

\begin{abstract}
RESUMO
O objetivo desse estudo foi avaliar o conhecimento de estudantes de Odontologia acerca do diagnóstico e da notificação em casos de violência contra crianças e adolescentes (VCA). Trata-se de um estudo transversal, descritivo e exploratório realizado com os estudantes $(n=100)$ que cursavam oitavo e décimo períodos no semestre 2018.1, dos turnos diurno e noturno do curso de Odontologia do Nordeste brasileiro. Os dados foram coletados por meio de questionário autoaplicável, composto por 22 perguntas. Os dados foram tabulados e analisados segundo estatística descritiva e teste de associação entre as variáveis, considerando p-valor <0,05 como significância estatística. A importância do tema foi reconhecida pela quase totalidade dos estudantes $(99,0 \%)$, no entanto, menos da metade $(45,0 \%)$ considerou que as informações recebidas na graduação foram suficientes. Destaca-se, porém, que os estudantes demonstraram conhecimento razoável do assunto, com percentual de acertos de 75,27\%. Não houve diferença significativa entre o número de acertos dos alunos do oitavo e décimo períodos. Embora 85,0\% dos estudantes afirmaram que a conduta correta em caso suspeito de VCA seja fazer denúncia ao Conselho Tutelar da localidade, menos da metade afirmou conhecer a ficha de notificação específica e apenas 10,0\% conheciam que a pena para os profissionais que não notificarem é multa de 3 a 20 salários de referência. Concluise que os estudantes apresentaram conhecimento satisfatório no que diz respeito ao diagnóstico de VCA e domínio sobre os meios de denúncia para notificação específica.
\end{abstract}

Descritores: Maus-tratos Infantis. Notificação Compulsória. Estudantes de Odontologia.

\section{INTRODUÇÃO}

A violência contra crianças e adolescentes constitui um grave problema de saúde pública e apresenta números alarmantes e crescentes, principalmente nas últimas décadas. No Brasil, entre 1980 e 2014, o número de homicídios de 
crianças e adolescentes cresceu 476,4\% $\%^{1,2}$. Outras formas de violência que não levam a óbito, como a física e sexual, são igualmente preocupantes, sendo a negligência a mais prevalente na população infantil ${ }^{3}$.

Crianças e adolescentes compõem o grupo mais exposto e vulnerável a sofrer violações de seus direitos, afetando direta e indiretamente sua saúde física, mental e emocional. Estudos epidemiológicos e sociológicos mostram que, frequentemente, as crianças são vítimas da violência desde o nascimento, inicialmente com predomínio de vítimas do sexo masculino, crianças menores de 5 anos vítimas de abandono e negligência, tendo os pais como agressores e a residência como o principal local de perpetração. Com o aumento da idade, tem-se a escola como local de risco e as vias públicas para meninos. $\mathrm{Na}$ fase da adolescência, os jovens aparecem também como agentes agressores, mas, sobretudo, como vítimas $^{3,4}$

Com a promulgação do Estatuto da Criança e do Adolescente (ECA) em 1990, a regulamentação específica sobre o assunto destaca o papel dos profissionais de saúde e de educação quanto à identificação e notificação de casos de violência infantil ${ }^{5}$. Em 2001, o Ministério da Saúde torna obrigatório para todas as instituições de saúde pública e/ou conveniadas ao Sistema Único de Saúde (SUS) em todo o território nacional, o preenchimento da Ficha de Notificação Compulsória e seu encaminhamento aos órgãos competentes ${ }^{6}$. Dando sequência à implementação dessas políticas e ações, o Ministério da Saúde lança em 2010 um documento pedagógico com o propósito de sensibilizar e orientar os gestores e profissionais de saúde para uma ação contínua e permanente para a atenção integral à saúde de crianças, adolescentes e suas famílias em situação de violências ${ }^{4}$.

Desde a implementação do ECA até o presente momento, percebem-se os avanços das políticas de saúde dirigidas às crianças nos indicadores de saúde, a exemplo da redução da mortalidade infantil. Porém, a violência contra crianças e adolescentes continua sendo uma prática comum e o número de notificações referentes à violência infantil no país é escasso ${ }^{4,7}$. Esse fato torna-se preocupante enquanto as informações estatísticas são essenciais para a elaboração de políticas de atendimento consistentes e comprometidas com a realidade da violência no país. Além disso, a notificação sendo obrigatória constitui-se num instrumento fundamental para impor medidas que visem cessar a violência no caso particular ${ }^{8,9}$.

Estudos demonstram que a maioria dos ferimentos decorrentes dos maus-tratos infantis envolve a região orofacial: cabeça, face, boca e pescoço. Além disso, pais de crianças vítimas de abuso frequentemente trocam de médico para evitar a detecção da violência, mas preferem levar os filhos para serem examinados pelo mesmo cirurgião-dentista (CD). Estes aspectos colocam o $\mathrm{CD}$ em uma posição oportuna para identificar essas vítimas. No entanto, muitos destes profissionais não têm o hábito de denunciar ou registrar casos suspeitos de agressão infantil e um dos principais motivos é a falta de capacitação ${ }^{10,11}$.

A resolução CNE/CES 312, de 19 de fevereiro de 2002, apresenta as Diretrizes Curriculares Nacionais (DCN) do Curso de Graduação em Odontologia ${ }^{12}$. O artigo $5^{\circ}$ diz que o Curso de Graduação em Odontologia tem como perfil do formando egresso/profissional o $\mathrm{CD}$ que respeite os princípios éticos inerentes ao exercício profissional e atue em todos os níveis de atenção à saúde, integrando-se em programas de promoção, manutenção, prevenção, proteção e recuperação da saúde, sensibilizados e comprometidos com o ser humano, respeitando-o e valorizando-o. Ainda sobre o perfil dos 
profissionais, o artigo aponta o exercício da profissão de forma articulada ao contexto social, entendendo-a como uma forma de participação e contribuição social.

Em consonância com as DCN, o tema da violência na formação do CD é importante para o fortalecimento de uma Odontologia mais eficiente e socialmente justa. Porém, muitos estudos ainda apontam para uma deficiência na formação profissional que capacite o $\mathrm{CD}$ para a imediata identificação e relato de casos de maustratos infantis ${ }^{7,13,14}$.

A atuação destes profissionais é fundamental e compreende a observação, registro e denúncia dos casos suspeitos às agências de proteção à criança. Desta forma, o objetivo do estudo foi avaliar o conhecimento dos estudantes do curso de Odontologia da Universidade federal de Pernambuco (UFPE) acerca do diagnóstico e da notificação de casos de violência contra crianças e adolescentes.

\section{METODOLOGIA}

Esse estudo, caracterizado como exploratório, descritivo, quantitativo, de corte transversal, foi realizado no curso de Odontologia da UFPE no ano de 2018. O projeto foi submetido e aprovado pelo Comitê de Ética em Pesquisa com Seres Humanos do Centro de Ciências da Saúde da UFPE (CAAE 69665817.5.0000.5208, parecer 2.576.115).

A população foi composta por estudantes cursando o $8^{\circ}$ e o $10^{\circ}$ períodos, durante o período de 2018/1, de ambos os sexos, que estavam devidamente matriculados no curso de Odontologia. Um único avaliador realizou a aplicação do questionário, após a leitura e assinatura do Termo de Consentimento Livre e Esclarecido. A aplicação foi realizada em sala de aula após uma rápida explicação sobre a finalidade da pesquisa e teve duração de cerca de 30 minutos.
$\mathrm{O}$ instrumento de pesquisa consistiu de um questionário (figura 1) de autopreenchimento, composto por 22 perguntas objetivas e uma única pergunta subjetiva. $\mathrm{O}$ instrumento foi elaborado com perguntas adaptadas do questionário de Kirankumar et al. $(2011)^{15}$ a respeito do diagnóstico de violência contra crianças e adolescentes. Além disso, foram elaboradas questões sobre dados do estudante, como idade e gênero, assim como sua percepção sobre a importância do tema na graduação, contato com o tema, suspeita de casos de violência na graduação, autoavaliação sobre a capacidade de notificar, procedimentos de denúncia e papel do $\mathrm{CD}$ quanto à conduta correta.

Os dados foram tabulados e analisados utilizando o software SPSS 20.0 (IBM, Armonk, NY, EUA). Os dados foram expressos como média, mediana, desvio-padrão, mínimo e máximo para as variáveis quantitativas e frequência absoluta e relativa para as variáveis qualitativas. Para verificar se houve associações com as variáveis qualitativas foi usado o teste Exato de Fisher e teste da razão de Verossimilhança. Para comparação de médias das variáveis contínuas foi realizado o teste não paramétrico de Mann-Whitney. A significância estatística de todos os testes foi considerada como p-valor $<0,05$.

\section{RESULTADOS}

A tabela 1 apresenta os dados de idade, sexo e período do curso. Observou-se predominância do sexo feminino, com faixa etária de 21 a 25 anos, com o menor percentual $(8,0 \%)$ na faixa etária de 30 anos ou mais, sem diferença significativa.

Os estudantes apresentarem um percentual médio de acertos de 75,27 $\pm 9,44 \%$ variando de $46,67 \%$ a $100,00 \%$ e não houve diferença estatisticamente significativa entre os alunos do $8^{\circ}$ e $10^{\circ}$ período $(\mathrm{p}$-valor $=0,113)($ tabela 2$)$. 


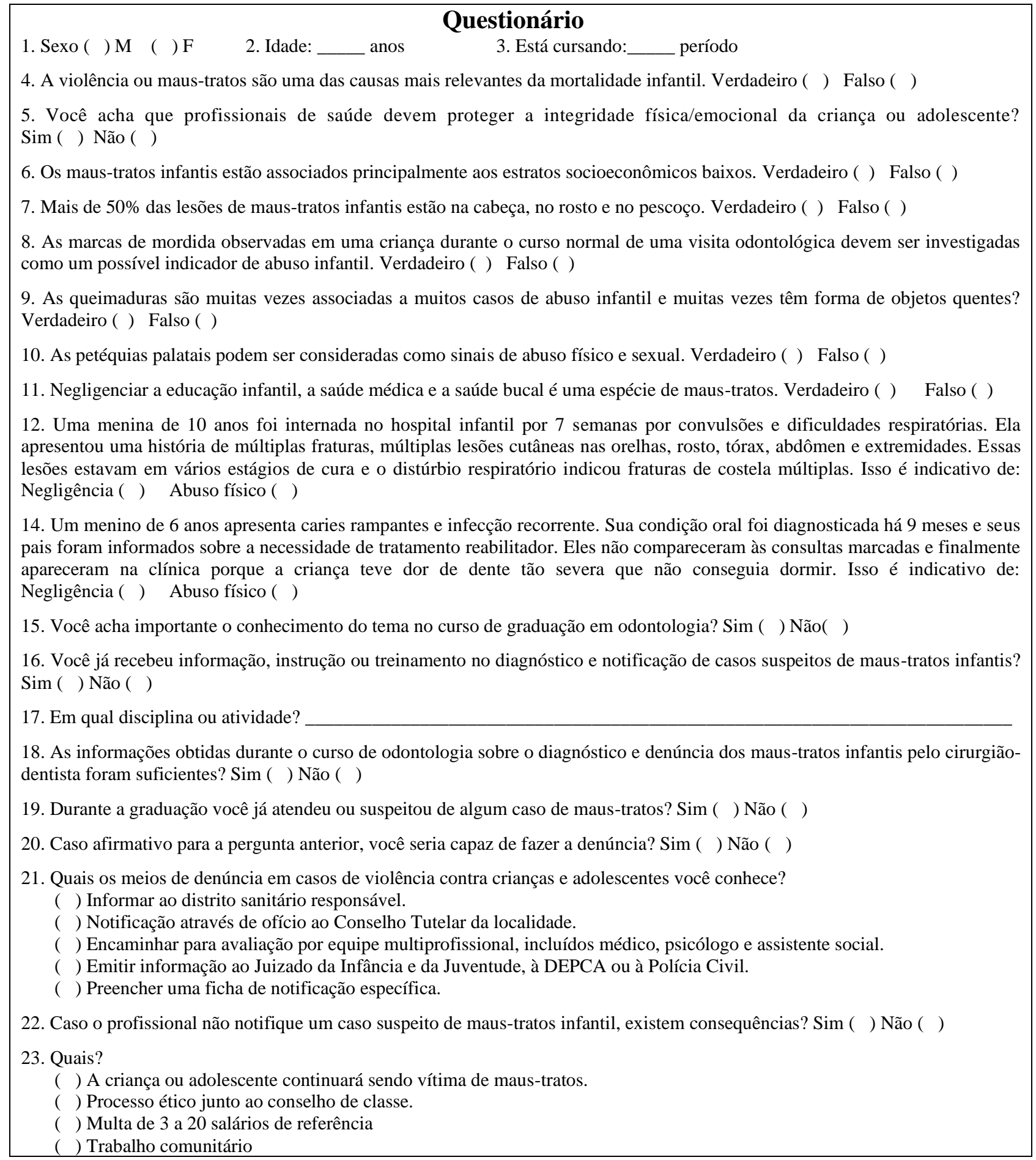

Figura 1. Questionário aplicado aos estudantes 
Tabela 1. Distribuição dos participantes da pesquisa por período letivo, segundo as variáveis sexo e faixa etária

\begin{tabular}{lccccccc}
\hline Variáveis & \multicolumn{9}{c}{ Período } & \multicolumn{3}{c}{ Total } & p-valor \\
& & $\mathbf{8}^{\mathbf{0}}$ & & $\mathbf{1 0}^{\mathbf{0}}$ & & & \\
Sexo & & $\mathbf{\%}$ & $\mathbf{n}$ & $\mathbf{\%}$ & $\mathbf{n}$ & $\mathbf{\%}$ & \\
$\quad$ Feminino & 34 & 66,7 & 31 & 63,3 & 65 & 65,0 & 0,721 \\
$\quad$ Masculino & 17 & 33,3 & 18 & 36,7 & 35 & 35,0 & \\
\hline Faixa Etária (anos) & & & & & & & \\
De 21 a 25 & 41 & 80,4 & 35 & 71,4 & 76 & 76,0 & 0,486 \\
De 26 a 30 & 6 & 11,8 & 10 & 20,4 & 16 & 16,0 & \\
Acima de 30 & 4 & 7,8 & 4 & 8,2 & 8 & 8,0 & \\
\hline Total & $\mathbf{5 1}$ & $\mathbf{1 0 0}$ & $\mathbf{4 9}$ & $\mathbf{1 0 0 , 0}$ & $\mathbf{1 0 0}$ & $\mathbf{1 0 0 , 0}$ & \\
\hline
\end{tabular}

Tabela 2. Medidas descritivas do percentual de acertos de todas as questões com gabarito entre os alunos do $8^{\circ}$ e $10^{\circ}$ períodos

\begin{tabular}{lcccccc}
\hline Período & n & Média & Desvio-padrão & Mínimo & Máximo & p-valor \\
\hline $8^{\mathbf{o}}$ & 51 & 76,34 & 8,98 & 46,67 & 86,67 & 0,113 \\
$10^{\circ}$ & 49 & 74,15 & 9,87 & 46,67 & 100,00 & \\
\hline Total & $\mathbf{1 0 0}$ & $\mathbf{7 5 , 2 7}$ & $\mathbf{9 , 4 4}$ & $\mathbf{4 6 , 6 7}$ & $\mathbf{1 0 0}$ & \\
\hline
\end{tabular}

Os resultados expressos em percentual de acertos nas perguntas sobre o diagnóstico de maus-tratos são apresentados na tabela 3. A maioria dos estudantes respondeu corretamente às perguntas, com percentual de acertos acima de $80,0 \%$. No entanto, apenas $50 \%$ da amostra considerou que a VCA não está associada aos estratos socioeconômicos mais baixos. É possível observar também que a proporção de percentual de acertos dos alunos do oitavo período foi um pouco maior, quando comparado com os do décimo período, embora sem diferença estatística. A exceção foi a questão sobre petéquias palatais, na qual os alunos do décimo período $(87,7 \%)$ tiveram uma taxa de acerto maior, quando comparado aos do oitavo período (86,3\%). Além disso, a pergunta sobre negligência infantil apresentou percentual de acertos de $98,0 \%$ para ambos os períodos.
A conduta dos acadêmicos frente à detecção dos maus-tratos infantis, bem como as consequências quando não há notificação por parte do profissional, é apresentada na mesma tabela. A maior parte dos pesquisados respondeu que a notificação deveria ser feita por meio de ofício ao Conselho Tutelar (85\%), porém cerca de metade destes não afirmou ser obrigatório o preenchimento de uma ficha de notificação específica $(44,0 \%)$. No que diz respeito a não notificação de casos suspeitos por parte dos profissionais, todos os alunos do oitavo período concordam que haveria consequências caso a notificação não fosse realizada, e apenas três alunos do décimo período não concordaram com a assertiva $(6,7 \%)$. Dentre as consequências caso não haja notificação, apenas às duas primeiras assertivas foram mais selecionadas. A alternativa referente 
à multa de 3 a 20 salários foi marcada apenas por três alunos do oitavo período $(5,9 \%)$, e sete alunos do décimo período $(14,3 \%)$.

Na tabela 4 estão os resultados relativos à abordagem do tema da violência contra crianças e adolescentes, informações sobre notificação, atendimento suspeito e capacidade de denúncia.
A maioria dos estudantes afirmou ter recebido informações ou instrução para o diagnóstico de maus-tratos, porém consideram as informações insuficientes $(55,0 \%)$. Como experiência, 15 alunos do oitavo período $(19,6 \%)$ atenderam casos com suspeita de maus-tratos, e 20 no décimo período $(30,6 \%)$.

Tabela 3. Distribuição da proporção percentual de acertos dos alunos do $8^{\circ}$ e $10^{\circ}$ para as perguntas a respeito do conhecimento geral e diagnóstico de violência contra crianças e adolescentes

\begin{tabular}{|c|c|c|c|c|c|c|c|}
\hline \multirow{3}{*}{$\begin{array}{l}\text { Questões } \\
\text { (conhecimento) }\end{array}$} & \multicolumn{4}{|c|}{ Período } & \multirow{2}{*}{\multicolumn{2}{|c|}{ Total }} & \multirow{3}{*}{ p-valor } \\
\hline & \multicolumn{2}{|c|}{$\mathbf{8}^{\mathbf{o}}$} & \multicolumn{2}{|c|}{$10^{\circ}$} & & & \\
\hline & $\mathbf{n}$ & $\%$ & $\mathbf{n}$ & $\%$ & $\mathbf{n}$ & $\%$ & \\
\hline \multicolumn{8}{|l|}{ Conhecimento de diagnóstico } \\
\hline Violência como causa de mortalidade infantil & 44 & 86,3 & 42 & 85,7 & 86 & 86,0 & 1,000 \\
\hline $\begin{array}{l}\text { Não há associação da violência com nível } \\
\text { socioeconômico baixo }\end{array}$ & 24 & 47,1 & 19 & 38,8 & 43 & 43,0 & 0,426 \\
\hline $\begin{array}{l}\text { Mais de } 50 \% \text { das lesões de maus-tratos infantis } \\
\text { ocorrem na cabeça, no rosto e no pescoço }\end{array}$ & 41 & 80,4 & 39 & 79,6 & 80 & 80,0 & 1,000 \\
\hline $\begin{array}{l}\text { Marcas de mordida como indicador de abuso } \\
\text { infantil }\end{array}$ & 48 & 94,1 & 46 & 93,9 & 94 & 94,0 & 1,000 \\
\hline Queimaduras como indicador de abuso infantil & 49 & 96,1 & 46 & 93,9 & 95 & 95,0 & 0,675 \\
\hline $\begin{array}{l}\text { Petéquias palatais como sinais de abuso físico e } \\
\text { sexual }\end{array}$ & 44 & 86,3 & 43 & 87,8 & 87 & 87,0 & 1,000 \\
\hline $\begin{array}{l}\text { Identificação de negligência de cuidados médicos e } \\
\text { educacionais como maus-tratos }\end{array}$ & 50 & 98,0 & 48 & 98,0 & 98 & 98,0 & 1,000 \\
\hline Identificação de sinais de abuso físico & 44 & 86,3 & 38 & 77,6 & 82 & 82,0 & 0,304 \\
\hline Identificação de sinais de negligência odontológica & 50 & 98,0 & 46 & 93,9 & 96 & 96,0 & 0,357 \\
\hline \multicolumn{8}{|l|}{ Conhecimento de denúncia } \\
\hline Informar ao distrito sanitário responsável & 13 & 25,5 & 10 & 20,4 & 23 & 23,0 & 0,637 \\
\hline $\begin{array}{l}\text { Notificação através de ofício ao Conselho Tutelar } \\
\text { da localidade }\end{array}$ & 42 & 82,4 & 43 & 87,8 & 85 & 85,0 & 0,578 \\
\hline $\begin{array}{l}\text { Encaminhar para avaliação por equipe } \\
\text { multiprofissional }\end{array}$ & 29 & 56,9 & 38 & 77,6 & 67 & 67,0 & 0,034 \\
\hline $\begin{array}{l}\text { Informar ao Juizado da Infância e da Juventude, à } \\
\text { DEPCA* ou à Polícia Civil }\end{array}$ & 31 & 60,8 & 26 & 53,1 & 57 & 57,0 & 0,545 \\
\hline $\begin{array}{l}\text { Consequências em casos de ausência de notificação } \\
\text { pelo profissional }\end{array}$ & 51 & 100,0 & 46 & 93,9 & 97 & 97,9 & 0,114 \\
\hline $\begin{array}{l}\text { A criança ou adolescente continuará sendo vítima } \\
\text { de maus-tratos }\end{array}$ & 47 & 92,2 & 42 & 85,7 & 89 & 89,9 & 0,352 \\
\hline Processo ético junto ao conselho de classe & 35 & 68,6 & 32 & 65,3 & 67 & 67,0 & 0,832 \\
\hline Multa de 3 a 20 salários de referência & 3 & 5,9 & 7 & 14,3 & 10 & 10,0 & 0,196 \\
\hline
\end{tabular}

*DEPCA - Delegacia Especializada de Proteção à Criança e ao Adolescente 
Tabela 4. Distribuição dos estudantes por período do curso com relação a valorização, formação e experiência com situações de suspeita de violência contra crianças e adolescentes

\begin{tabular}{|c|c|c|c|c|c|c|c|}
\hline \multirow{3}{*}{ Questões } & \multicolumn{4}{|c|}{ Período } & \multirow{2}{*}{\multicolumn{2}{|c|}{ Total }} & \multirow{3}{*}{ p-valor } \\
\hline & \multicolumn{2}{|c|}{$\mathbf{8}^{\mathbf{o}}$} & \multicolumn{2}{|c|}{$10^{\mathbf{o}}$} & & & \\
\hline & $\mathbf{n}$ & $\%$ & $\mathbf{n}$ & $\%$ & $\mathbf{n}$ & $\%$ & \\
\hline $\begin{array}{l}\text { Profissionais de saúde devem proteger a } \\
\text { integridade da criança/adolescente }\end{array}$ & 51 & 100,0 & 49 & 100,0 & 100 & 100,0 & - \\
\hline $\begin{array}{l}\text { O tema da violência é importante para a } \\
\text { formação de cirurgiões-dentistas }\end{array}$ & 50 & 98,0 & 49 & 100,0 & 99 & 99,0 & 1,000 \\
\hline $\begin{array}{l}\text { Recebeu instrução sobre o diagnóstico e } \\
\text { notificação }\end{array}$ & 47 & 92,2 & 44 & 89,8 & 91 & 91,0 & 0,738 \\
\hline $\begin{array}{l}\text { Obteve informações adequadas durante } \\
\text { o curso de graduação }\end{array}$ & 21 & 41,2 & 24 & 49,0 & 45 & 45,0 & 0,547 \\
\hline $\begin{array}{l}\text { Teve experiência com diagnóstico ou } \\
\text { suspeita de maus-tratos durante o } \\
\text { atendimento de crianças e adolescentes }\end{array}$ & 10 & 19,6 & 15 & 30,6 & 25 & 25,0 & 0,251 \\
\hline Sente-se capaz de efetuar a denúncia & 9 & 90,0 & 14 & 93,3 & 23 & 92,0 & 1,000 \\
\hline
\end{tabular}

\section{DISCUSSÃO}

De acordo com o Ministério da Saúde, a violência contra crianças e adolescentes é o grande desafio do século e incorre em altos custos econômicos e sociais tanto para o governo federal como para as famílias ${ }^{4}$. Dados do Sistema de Informação sobre Mortalidade (SIM), no ano de 2014, apontam que as causas externas (acidentes e violência) foram os principais motivos de mortalidade em crianças de 1 a $10 \operatorname{anos}^{15}$. O presente estudo mostra que quase $90,0 \%$ dos alunos reconhecem a violência como uma das causas mais relevantes da mortalidade infantil. Bodrumlu et al. (2018) ${ }^{17}$, em um estudo realizado com estudantes da Turquia, observaram que apenas $22,14 \%$ dos alunos entrevistados observaram esta associação.

$\mathrm{Na}$ amostra do presente estudo houve predominância de estudantes do gênero feminino (66,7\%), sendo a maioria com idade entre 21 e 25 anos $(80,4 \%)$. Essa distribuição também foi observada em um estudo realizado por Sousa et al. $(2012)^{18}$, com 59 acadêmicos, no curso de graduação em Odontologia da UNIPÊ em João Pessoa.
Embora a violência seja um fenômeno multicausal que ocorre em toda a sociedade sem distinção de nível socioeconômico, a violência que ocorre em famílias com maior poder socioeconômico é menos visível, o que faz com que profissionais de saúde associem a violência doméstica às famílias mais pobres ${ }^{19}$. A maioria dos estudantes entrevistados associaram a violência aos estratos socioeconômicos mais baixos, resultados também observados por Jundi et al. $(2017)^{20}$ em pesquisa com estudantes de pós-graduação em duas escolas de Odontologia da Jordânia. O estudo realizado por Bodrumlu et al. $(2018)^{17}$ observou que $25,26 \%$ dos alunos estavam cientes de que o abuso infantil pode ocorrer entre pessoas de alta renda.

Alguns estudos mostram que o conhecimento acerca do tema não é o esperado, pois um percentual considerável de estudantes e profissionais ainda não estão cientes da alta prevalência de tais lesões, bem como não sabem qual a região do corpo mais afetada em casos de violência infantil ${ }^{14,20,21}$. Neste sentido, o resultado do presente estudo surpreende apontando um percentual elevado de acertos $(75,27 \%)$ e a 
maioria das questões com acertos entre 80 e $90 \%$. Destaca-se que o tema é abordado em disciplina que ocorre no $7^{\circ}$ período do curso de Odontologia na UFPE. Supõe-se que, sendo a violência um tema recente na agenda da saúde ${ }^{4}$ o conhecimento dos profissionais de saúde tende a melhorar com os avanços das políticas e ações decorrentes.

É possível observar que quase $95,0 \%$ dos estudantes concordam que as queimaduras estão presentes em muitos casos de abuso infantil, assim como, o mesmo resultado é obtido para reconhecer que as marcas de mordidas são frequentemente um componente de abuso infantil. Os dados encontrados são semelhantes aos resultados encontrados por Hashim e Al-Ani (2013) ${ }^{22}$.

Os CD podem desempenhar um papel importante na identificação e notificação de casos de abuso físico infantil. Na verdade, podem ser o primeiro profissional a notar casos de abuso físico, visto que 50 a $65 \%$ das lesões ocorrem na região de cabeça e pescoço, sendo muitas vezes lesões orofaciais, regiões que eles rotineiramente costumam examinar ${ }^{23,24,25}$. Foi observado que a maior parte dos estudantes $(80,0 \%)$ tinha o conhecimento de que mais da metade das lesões de maus-tratos infantis estão localizadas na cabeça, no rosto e no pescoço. Este resultado foi mais satisfatório do que o encontrado por Wacheski et al. (2012) ${ }^{26}$, em Curitiba, no qual $59,0 \%$ dos estudantes identificam a região de cabeça e pescoço como sendo a região do corpo mais atingida e também semelhante aos achados do estudo de Massoni et al. $(2010)^{10}$.

A violência sexual muitas vezes é imposta à criança ou ao adolescente em relações intra ou extrafamiliar, por força física ou psicológica. $\mathrm{O}$ CD deve estar atento a sinais de abuso sexual, que podem ser identificados pela presença de lesões ou sinais de doenças sexualmente transmissíveis na cavidade bucal. Este foi tema de uma das questões presente no estudo de Wacheski et al.
$(2012)^{26}$, onde grande parte dos estudantes não respondeu adequadamente antes ou mesmo após a cartilha informativa, revelando dificuldades no assunto. Porém, relacionando abuso sexual com sinais na boca, no presente estudo, foi observado que quase $90,0 \%$ concordaram que as petéquias palatais podem ser consideradas como sinais de abuso físico e sexual.

A maioria dos estudantes $(98,0 \%)$ concordam que negligenciar a educação infantil, a saúde médica e a saúde bucal é uma espécie de maus-tratos e segundo o Comitê da Academia Americana de Pediatria sobre Abuso e Negligência Infantil e a Academia Americana de Odontopediatria, a falta de condições básicas para ao desenvolvimento da criança é considerada negligência, sendo que a desatenção quanto à saúde bucal da criança também pode ser caraterizada como negligência ${ }^{28}$. No Brasil, o Art. 227 da Constituição Federal, de 1988 diz: "É dever da família, da sociedade e do Estado assegurar à criança e ao adolescente, com absoluta prioridade, o direito à vida, à saúde, à alimentação, ao lazer, à profissionalização, à cultura, à dignidade, ao respeito, à liberdade e à convivência familiar e comunitária, além de colocá-los a salvo de toda forma de negligência, discriminação, exploração, violência, crueldade e opressão"29.

Mais de 80,0\% dos estudantes de ambos os períodos souberam identificar o tipo de violência, no caso violência física e negligência, de acordo com as características citadas. Massoni et al. $(2010)^{10}$ revelam que o diagnóstico de maustratos à criança deve ser focado não apenas nos ferimentos traumáticos, mas também no comportamento da criança e dos pais. Já com relação à negligência, segundo Granville-Garcia et al. $(2015)^{30}$ existe a falta de conhecimento dos pais e responsáveis quanto à conduta negligente contra as crianças, as quais são consideradas incapazes e necessitam receber cuidados básicos, 
conforme o ordenamento jurídico nacional.

Segundo o Conselho Federal de Odontologia, é dever do cirurgião-dentista zelar pela saúde e dignidade do paciente ${ }^{31}$. Todos os estudantes entrevistados concordam que o profissional deve ter responsabilidade ética em proteger as crianças da violência. Resultados similares foram encontrados nos estudos feitos por Al-Jundi et al. $(2017)^{19}$ e Hashim e Al-Ani $(2013)^{27}$. Além disso, os estudantes acham importante o tema no curso $(99,0 \%)$. Um estudo de Silva Jr et al. $(2015)^{31}$, feito no curso de Odontologia da UFES, no semestre letivo de 2010/2, observou-se que dos 100 estudantes entrevistados, apenas dois não achavam importante o tema no curso e dois não sabiam responder.

Outros estudos mostram que os estudantes de Odontologia recebem informação sobre o assunto durante a graduação ${ }^{14,18,33}$, porém o tema é abordado principalmente no final do curso, nas disciplinas de Odontopediatria, provavelmente por ser o momento de maior atenção a crianças e adolescentes, confirmando os dados obtidos na presente pesquisa. El Sarraf et al. (2012) ${ }^{33}$ verificaram que $88 \%$ dos profissionais pesquisados tiveram pouca informação sobre o assunto na graduação, contradizendo os estudos citados anteriormente. Da mesma forma, a maioria dos estudantes entrevistados em estudos realizados por Jordan et al. $(2012)^{21}$ e Silva Jr et al. (2015) ${ }^{32}$ informou não ter obtido informações sobre maus-tratos infantis durante a graduação.

Observou-se que os estudantes consideram que as informações obtidas durante a graduação não foram suficientes. Resultados semelhantes foram encontrados nos estudos realizados por Serpa e Ramos $(2011)^{35}$ e Sousa et al. $(2012)^{18}$, nos quais $90,0 \%$ dos estudantes consideraram insuficientes as informações recebidas. Outros estudos mostram também a necessidade de ampliar o conhecimento dos CD sobre o tema, devido à baixa notificação de casos suspeitos de maus-tratos ${ }^{34,36}$.

Quanto à experiência vivenciada pelos estudantes é possível observar que $25,0 \%$ atenderam casos de suspeita de maus-tratos, e apenas um estudante de cada período não se julgou capaz de fazer a denúncia. Resultados diferentes foram encontrados em um estudo realizado por Abreu et al. $(2017)^{36}$, quando os estudantes alegaram não ter se deparado com casos suspeitos de abuso ou de traumas orofaciais que pudessem ter relação com maus-tratos infantis e a grande maioria $(95,0 \%)$ nunca viu algum caso de suspeita de abuso físico entre seus pacientes.

Em relação aos meios de denúncia, a notificação por meio de ofício ao Conselho Tutelar da localidade foi a opção mais marcada pela maioria dos entrevistados, porém, somente metade deles tinham o conhecimento sobre o preenchimento de uma ficha de notificação específica. $\mathrm{O}$ presente estudo apresenta similaridade com diversas pesquisas ${ }^{18,22,35,37}$. Porém, o resultado obtido não é o mesmo encontrado nas pesquisas feitas por Al-Jundi et al. (2012) $)^{20}$; Jordan et al. (2012) ${ }^{21}$; Mogaddam et al. $(2016)^{37}$, nas quais os resultados encontrados mostraram que quase dois terços da população do estudo não sabia onde relatar casos suspeitos de abuso infantil.

Ressalta-se que, na falta do Conselho Tutelar no município, recomenda-se notificar ao juizado da infância e da juventude, aos órgãos de proteção à criança, ou ainda reportar o caso às autoridades policiais e solicitar o encaminhamento para o exame de corpo de delito $^{5,39}$. O profissional que não tenha acesso a notificação feita através do Conselho Tutelar deve buscar outros meios que assegurem à criança ou ao adolescente a denúncia da condição de maus-tratos. Por isso, para uma melhor avaliação do conhecimento dos estudantes, outras opções foram oferecidas e assinaladas em maior 
ou menor proporção pelos estudantes. Treze alunos do oitavo e dez do décimo período concordaram que a suspeita de maus-tratos deveria ser informada ao distrito sanitário responsável, assim como mais de $50 \%$ dos estudantes do oitavo e décimo períodos marcaram a opção de encaminhar para avaliação por equipe multiprofissional, incluindo Médico, Psicólogo e Assistente Social. Mais da metade dos alunos de ambos os períodos concordaram com a opção de emitir informação ao Juizado da Infância e da Juventude, à DEPCA ou à Polícia Civil. Um estudo feito por Gomes et al. (2011) ${ }^{39}$ com alunos da UEPB de João Pessoa/PB verificou que 83,3\% dos acadêmicos denunciariam ao Conselho Tutelar, 29,6\% questionariam os pais e 9,3\% indicariam algum tratamento psicológico, dentre outras atitudes.

A notificação de maus-tratos é o processo que visa interromper as atitudes $\mathrm{e}$ comportamentos por parte do agressor, e deve ser realizada ao Conselho Tutelar da respectiva localidade da vítima. O Conselho Tutelar, quando informado, irá analisar o caso e encaminhar soluções. De acordo com o Estatuto da Criança e Adolescente, a não notificação em caso de suspeita de maus-tratos, pode acarretar pena de 3 a 20 salários mínimos, e o dobro em caso de reincidência ${ }^{4,10,25,32,39}$. Todos os alunos do oitavo período e 97,0\% do décimo período concordaram que existirá consequência caso não haja notificação por parte do profissional. A opção de que a criança ou adolescente continuará sendo vítima de maus-tratos foi a mais assinalada pelos estudantes de ambos os períodos, seguida de processo ético junto ao conselho de classe. Porém, a multa de 3 a 20 salários de referência, que seria a principal resposta da pergunta em questão, foi a alternativa menos escolhida.

O estudo possui limitações, tais como o uso de questionário de informações autodeclaradas e a falta de um questionário validado utilizado por outros autores. No entanto, a pesquisa não teve recusa de participantes, o que fortalece os resultados apresentados, os quais demonstram um conhecimento satisfatório por parte dos alunos de Odontologia acerca do diagnóstico e notificação de casos suspeitos ou confirmados de violência contra crianças e adolescentes.

É importante que os profissionais de saúde (incluindo os $\mathrm{CD}$ ) estejam cientes de que o abuso físico ou sexual pode resultar em lesões ou condições bucais ou dentárias. Também devem estar cientes de quando e como documentar lesões suspeitas e como obter evidências laboratoriais, documentação fotográfica e/ou consulta com especialistas quando apropriado ${ }^{28}$. Para tanto, é essencial que os profissionais sejam treinados desde a graduação e que haja investimento em educação continuada no tema da notificação das violências, estratégia preconizada na Política Nacional de Redução da Morbimortalidade por Acidentes e Violências ${ }^{41} \mathrm{e}$ na Política Nacional de Promoção da Saúde ${ }^{42}$, que priorizam a prevenção da violência e o estímulo à cultura da paz. Tais medidas visam fortalecer o sistema de vigilância no país com o aumento do número de notificações por profissionais de saúde, em um exercício de cidadania, cuidado e proteção da criança e do adolescente.

\section{CONCLUSÃO}

Não houve diferença significativa nas respostas obtidas pelos estudantes dos diferentes períodos. Porém, observou-se que a taxa de resposta foi maior entre estudantes do oitavo período, devido ao tempo de contato mais próximo com o assunto abordado. Os estudantes apresentaram conhecimento bastante satisfatório no que diz respeito ao diagnóstico de maus-tratos, e muitos suspeitaram de casos atendidos no âmbito da graduação. A grande maioria demonstrou domínio sobre os meios de denúncia para notificação nos casos de suspeita de maus- 
tratos. Apesar disso, poucos sabiam da existência de uma ficha de notificação específica e da consequência prevista em lei para os profissionais que não realizam a notificação de casos suspeitos ou confirmados de violência contra crianças e adolescentes. Com o avanço das políticas e ações decorrentes destas o conhecimento dos profissionais de saúde tende a melhorar.

\section{ABSTRACT \\ Diagnosis and reporting of cases of violence against children and adolescents: knowledge of dental students}

The aim of this study was to evaluate the knowledge of dental students about the diagnosis and notification in cases of violence against children and adolescents (VCA). This is a crosssectional, descriptive and exploratory study conducted with dental students $(\mathrm{n}=100)$ attending the eighth and tenth periods, between March and June 2018, at an undergraduate dental program in a northeastern Brazilian city. Data were collected through a self-administered questionnaire consisting of 22 questions. Data were tabulated and analyzed according to descriptive statistics and association test between variables, considering a p-value $<0.05$ as statistically significant. The importance of the topic was recognized by almost all students (99.0\%); however, less than half $(45.0 \%)$ considered that the information received during the undergraduate course was sufficient. It is noteworthy that the students demonstrated reasonable knowledge of the subject, with a percentage of correct answers of $75.27 \%$. There was no significant difference regarding the number of correct answers between the eighthand tenth-period students. Although $85.0 \%$ of students said that the correct conduct in case of suspected VCA is to report them to the local Guardianship Council, less than half said they knew the specific notification form and only $10.0 \%$ knew that the penalty for professionals who do not notify these cases is a fine ranging from 3 to 20 minimum wages. It was concluded that the students demonstrated satisfactory knowledge regarding the diagnosis of VCA and knew about the means of reporting these cases.

Descriptors: Child Abuse. Mandatory Reporting. Students, Dental.

\section{REFERENCIAS}

1. Waiselfisz JJ. Mapa da Violência 2012 crianças e adolescentes do brasil. $1^{\mathrm{a}}$ ed. Rio de Janeiro; 2012.

2. Waiselfisz JJ. Homicides of children and adolescents in Brazil. Homicide Dispatch 4. Rio de janeiro: Iguarapé Institute; 2017.

3. Malta DC, Bernal RTI, Teixeira BSM, Silva MMA, Freitas MIF. Fatores associados a violências contra crianças em Serviços Sentinela de Urgência nas capitais brasileiras. Cien Saude Colet. 2017;22(9):2889-98.

4. Brasil. Linha de Cuidado para a Atenção Integral à Saúde de Crianças, Adolescentes e suas Famílias em Situações de Violência. $1^{\text {a }}$ ed. Brasilia-DF: MS; 2010.

5. Brasil. Ministério da Saúde. Estatuto da Criança e do Adolescente, Brasília, 1990.

6. Brasil. Notificação de maus-tratos contra crianças e adolescentes: um passo a mais na cidadania em saúde. $2^{\mathrm{a}}$ ed. Brasília; 2002.

7. Assis SG, Avanci JQ, Pesce RP, Pires TO, Gomes DL. Notificações de violência doméstica, sexual e outras violências contra crianças no Brasil. Cien Saude Colet. 2012;17(9):2305-17.

8. Garbin CAS, Rovida TAS, Joaquim RC, Paula AM, Queiroz APDG. Violência denunciada: ocorrências de maus tratos contra crianças e adolescentes registradas em uma unidade policial. Rev Bras Enferm. 2011; 64(4):665-70.

9. Veloso MMX, Magalhães CMC, Dell'aglio DD, Cabral IR, Gomes MM. Notificação da violência como estratégia de vigilância em saúde: perfil de uma metrópole do Brasil. Cien Saude Colet. 2013;18(5):1263-72. 
10. Massoni ACLT, Ferreira ÂMB, Aragão AKR, Menezes VA, Colares V. Aspectos orofaciais dos maus-tratos infantis e da negligência odontológica. Cien Saude Colet. 2010;15(2):403-10.

11. Costacurta M, Benavoli D, Arcudi G, Docimo R. Oral and dental signs of child abuse and neglect. Oral Implantol (Rome). 2015:8(2-3):68.

12. Conselho Nacional de Educação/Câmara de Educação Superior (Brasil). Resolução $n^{\circ}$. 3, de 19 fevereiro de 2002. Diretrizes Curriculares Nacionais do Curso de Graduação em Odontologia. Diário Oficial da União 4 de março de 2002; Seção 1.

13. Luna GLM, Ferreira RC, Vieira LJES. Notificação de maus tratos em crianças e adolescentes por profissionais da Equipe de Saúde da Família. Cien Saude Colet. 2010;15(2):481-91.

14. Moura AR, Amorim A, Proença L, Milagre V. Dentists and undergraduate dental students require more information relating to child abuse. Medical Express. 2015;2(2): M150203.

15. Kirankumar SV, Noorani H, Shivprakash PK, Sinha S. Medical professional perception, attitude, knowledge, and experience about child abuse and neglect in Bagalkot district of north Karnataka: a survey report. J Indian Soc Pedod Prev Dent. 2011; 29(3):193-7.

16. Brasil. Ministério da Saúde. Uma análise da Situação de Saúde e das Causas externas. Saúde Brasil 2014. Brasília: MS; 2015.

17. Bodrumlu E, Avsar A, Arslan S. Assessment of knowledge and attitudes of dental students in regard to child abuse in Turkey. Eur J Dent Educ. 2018; 22: 40-6.

18. Sousa GFP, Carvalho MMP, GranvilleGarcia AF, Gomes MNC, Ferreira JMS. Conhecimento de acadêmicos em odontologia sobre maus-tratos infantis. Odonto. 2012;20(40):101-8.

19. Keenan HT, Campbell KA, Page K, Cook LJ, Bardsley T, Oslon LM. Perceived social risk in medical decision making for physical child abuse: a mixed methods study. BMC Pediatr. 2017;17:214.

20. Al-Jundi SHS, Zawaideh FI, Al-Rawi MH. Jordanian Dental students' knowledge and attitudes in regard to child physical abuse. $\mathrm{J}$ Dent Educ. 2010;74(10):1159-65.

21. Jordan A, Welbury RR, Tiljak MK. Croatian Dental Students' educational experiences and knowledge in regard to child abuse and neglect. J Dent Educ. 2012;76(11):1512-9

22. Fisher-Owens SA, Lukefahr JL, Tate AR, American Academy of Pediatrics, Section on Oral Health Committee on Child Abuse and Neglect, American Academy of Pediatric Dentistry, Council on Clinical Affairs, Council on Scientific Affairs, ad hoc work group on child abuse and neglect. Oral and Dental Aspects of Child Abuse and Neglect. Pediatrics. 2017;140(2) :20171487

23. UNICEF. (2009). Child abuse: A painful reality behind closed doors. Challenges: Newsletter on progress towards millennium development goals from a child rights. Perspective, 9, 1-12.

24. Cavalcanti AL. Prevalence and characteristics of injuries to the head and orofacial region in physically abused children and adolescents - a retrospective study in a city of the Northeast of Brazil. Dent Traumatol. 2010;26:149-53.

25. Valente LA, Dalledone M, Pizzatto E, Zaiter W, Souza JF, Losso EM. Domestic Violence against children and adolescents: prevalence of physical injuries in a southern Brazilian metropolis. Braz Dent J. 
2015;26(1):50-60.

26. Wacheski A, Lopes MGK, Paola APBP, Valença P, Losso EM. O conhecimento do aluno de Odontologia sobre maus tratos na infância antes e após o recebimento de uma cartilha informativa. Odonto. 2012;20(39): 7-15.

27. Hashim R, Al-Ani A. Child physical abuse: assessment of dental students' attitudes and knowledge in United Arab Emirates. Eur Arch Paediatr Dent. 2013; 14:301-05.

28. Brasil. Constituição da República Federativa do Brasil. Brasília-DF: Senado Federal; 1988.

29. Granville-Garcia AF, Vaz TMT, Martins VM, Massoni ACLT, Cavalcanti AL, Menezes VA. Maus tratos em crianças e adolescente de Solânea, Paraíba, Brasil: ocorrência e conduta profissional. Rev Bras Pesqui Saúde. 2010;12(4): 26-33.

30. Conselho Federal de Odontologia. Código de Ética Odontológica aprovado pela Resolução CFO-118/2012

31. Silva Júnior MF, Pagel MD, Campos DMKS, Miotto MHMBM. Conhecimento de acadêmicos de Odontologia sobre maustratos infantis. Arq Odontol. 2015;51(3):138-44.

32. Biss SP, Duda JG, Tomazinho PH, Pizzatto E, Losso EM. Maus tratos infantis: avaliação do currículo dos cursos em odontologia. Rev ABENO. 2015;15(1):5562.

33. El sarraf MC, Marengo G, Correr GM, Pizzatto E, Losso EM. Physical child abuse: perception, diagnosis, and management by southern Brazilian pediatric dentists. Pediatr Dent. 2012; 34(4): 72-6.

34. Serpa EM, Ramos AAS. Percepção dos maus tratos infantis pelos estudantes de odontologia da UFPB. Int $\mathrm{J}$ Dent. 2011:10(4):234-41.
35. Azevedo MS, Goettems ML, Brito A, Possebon AP, Domingues J, Demarco FF, Torriani DD. Child maltreatment: a survey of dentists in southern Brazil. Braz Oral Res. 2012;26(1):5-11.

36. Abreu PTR, Costa IFS, Galvão A, Souza ACPS, Zocratto KBF, Oliveira CAS. Abuso físico infantil: vivências e atitudes de estudantes de Odontologia. Rev ABENO. 2017;17(2):107-19.

37. Mogaddama M, Kamala I, Merdadc L, Alamoudi N. Knowledge, attitudes, and behaviors of dentists regarding child physical abuse in Jeddah, Saudi Arabia. Child Abuse Negl. 2016;54:43-56.

38. Matos FZ, Borges AH, Neto IM, Rezende CD, Silva KL, Pedro FLM, Porto AN. Avaliação do conhecimento dos alunos de graduação em odontologia $\mathrm{x}$ cirurgião dentista no diagnóstico de maus-tratos a crianças. Rev Odontol Bras Central. 2013;22(63).

39. Gomes LS, Pinto TCA, Costa EMMB, Ferreira JMSF, Cavalcanti SDLB. Granville-Garcia AF. Percepção dos acadêmicos de odontologia sobre maus tratos na infância. Odont Clín-Cient. 2011;10(1) $73-8$.

40. Brasil. Ministério da Saúde. Política Nacional de Redução de Morbimortalidade por Acidentes e Violência (PNRMAV). Brasília: Ministério da Saúde; 2002. 39

41. Brasil. Ministério da Saúde. Secretaria de Vigilância em Saúde. Política Nacional de Promoção da Saúde. Portaria $n^{\circ} 687$ MS/GM, de 30 de março de 2006. Brasília: MS; 2006.

\section{Correspondência para:}

Letícia Santos Alves de Melo

e-mail: leticia.melo41@gmail.com 
Diagnóstico e notificação de casos de violência contra crianças e adolescentes: conhecimento de estudantes de Odontologia

Rua Demócrito de Souza Filho, 156/1301

50610-120 Recife/PE 